\title{
Applicability of the 2001 revised diagnostic criteria in Brazilian Vogt-Koyanagi-Harada disease patients
}

\author{
Aplicabilidade do critério diagnóstico revisado de 2001 em pacientes \\ brasileiros com doença de Vogt-Koyanagi-Harada
}

\author{
Isabel Habeyche Cardoso ${ }^{1}$ \\ Moysés Eduardo Zajdenweber ${ }^{2}$ \\ Cristina Muccioli ${ }^{3}$ \\ Luciana Peixoto Fimamor ${ }^{4}$ \\ Rubens Belfort Jr. ${ }^{5}$
}

\begin{tabular}{l} 
ABSTRACT \\
\hline Purpose: To determine the applicability of the international revised \\
diagnostic criteria for Vogt-Koyanagi-Harada disease. Methods: Re- \\
trospective study. Medical charts of 140 patients with the diagnosis of \\
Vogt-Koyanagi-Harada disease, from the Uveitis Sector of the Federal \\
University of Sao Paulo(UNIFESP), were revised and classified following \\
the revised diagnostic criteria. Results: Of the 140 patients, $12.85 \%$ \\
fulfilled the criteria for complete disease, $29.28 \%$ incomplete disease, \\
$28.57 \%$ "probable" Vogt-Koyanagi-Harada disease and 28.27\% were con- \\
sidered not Vogt-Koyanagi-Harada disease. Conclusion: The authors \\
consider that the international revised diagnostic criteria have good \\
applicability and are very useful to helpin the diagnosis of Vogt-Koyanagi- \\
Harada disease.
\end{tabular}

Keywords: Uveomeningoencephalitis syndrome/diagnosis; Diagnostic techniques, ophthalmological; Uveitis; Granuloma

\footnotetext{
Trabalho realizado no setor de Úvea e AIDS da Universidade Federal de São Paulo - UNIFESP - São Paulo (SP) - Brazil.

Department of Ophthalmology. UNIFESP - São Paulo (SP) - Brazil.

2 Department of Ophthalmology. UNIFESP - São Paulo (SP) - Brazil. Department of Ophthalmology and Pathology, McGill University Hospital Center \& Henry C. Witelson Ocular Pathology Laboratory - Montreal Canadá.

Department of Ophthalmology. UNIFESP - São Paulo (SP) - Brazil.

${ }^{4}$ Department of Ophthalmology. UNIFESP - São Paulo (SP) - Brazil.

Department of Ophthalmology. UNIFESP - São Paulo (SP) - Brazil.

The authors have no financial conflict of interest regarding this manuscript.

Correspondence and reprint requests: Moysés E. Zajdenweber. Rua Desemb. Renato Tavares, 30/302 -

Rio de Janeiro (RJ) CEP 22411-060

E-mail: moysesz@uol.com.br

Recebido para publicação em 25.01.2007

Aprovação em 16.10.2007

Nota Editorial: Depois de concluída a análise do artigo sob sigilo editorial e com a anuência do Dr. Haroldo Vieira de Moraes Jr. sobre a divulgação de seu nome como revisor, agradecemos sua participação neste processo.
}

\section{INTRODUCTION}

Vogt-Koyanagi-Harada (VKH) disease is considered a bilateral, granulomatous panuveitis associated with central nervous system, auditory, and integumentary manifestations. It initially manifests with prodromal symptoms similar to aseptic meningitis, followed by posterior uveitis with exudative retinal detachments and disc hyperemia ${ }^{(1)}$. VKH disease is thought to be a T-cell-mediated autoimmune process directed against one or more antigenic components of melanocytes, a cell shared by the involved regions ${ }^{(2)}$. The disease occurs more frequently in pigmented groups, it is likely that certain ethnic groups have genetic backgrounds that predispose them to VKH disease. Specific human leucocyte antigen (HLA) types have been reported to be associated with the disease. Findings from different parts of the world indicate that there is an association between class II, subtype DR4 HLA and VKH disease, especially the HLA-DRB1*0405 allele ${ }^{(3)}$. The precise cause of the disease is uncertain, but T-lymphocytemediated autoimmunity directed against melanocytes is likely to play an important role ${ }^{(4)}$.

There are no specific diagnostic tests for VKH disease. At present, the diagnosis is usually made based on the clinical findings of prodromal CNS findings followed by a bilateral exudative posterior uveitis ${ }^{(5)}$. In addition the disease could have ocular and extraocular symptoms, only extraocular or only ocular symptoms.

The typical treatment for VKH disease is high-dose corticosteroid the- 
rapy followed by a slow tapering of the drug over 3 to 6 months. Patients who are intolerant to or with disease that is resistant to corticosteroid therapy may be treated with immunosuppressive drug therapy to minimize complications and to improve visual prognosis ${ }^{(6)}$. Intravitreal triamcinolone acetonide may provide short-term improvement in visual acuity and resolution of serous retinal detachments in patients with VKH disease ${ }^{(7)}$.

The first attempt to establish diagnostic criteria for $\mathrm{VKH}$ disease took place in 1978 at the Annual Meeting of the American Uveitis Society ${ }^{(8)}$. Because the sequential development of these findings often makes prospective studies unfeasible, the diagnostic criteria were revised by the First International Workshop on VKH disease in $1999^{(9)}(\text { Figure } 1)^{(9)}$.

The new criteria take into account the multisystemic nature of VKH disease, with allowance for the different ocular findings that are present in the early and late stages of the disease ${ }^{(3)}$.

In the present study the authors reviewed charts of patients with a previous diagnosis of Vogt-Koyanagi-Harada disease assessing the reviewed diagnostic criteria, to check the applicability of the revised criteria in a Brazilian VKH disease population.

\section{METHODS}

Medical charts of 140 patients with a diagnosis of $\mathrm{VKH}$ disease, from the Uveitis Sector of the Federal University of
Sao Paulo (UNIFESP), were revised and classified according to the 2001 revised diagnostic criteria (Figure 1) ${ }^{(9)}$.

A questionnaire based on the "VKH Syndrome - First International Workshop, 1999" was filled out by the authors, according to the information obtained from the charts.

All the patients with the diagnosis of VKH disease were included in the study.

\section{RESULTS}

Of the total of 140 patients diagnosed as having $\mathrm{VKH}$ disease, $18(12.85 \%)$ fulfilled the criteria for complete disease, $41(29.28 \%)$ incomplete disease, $40(28.57 \%)$ "probable" VKH (only ocular disease) and $40(28.27 \%)$ were considered not having VKH disease (Figure 2), according to the 2001 revised diagnostic criteria (Figure 1) ${ }^{(9)}$.

Forty-one patients were diagnosed as having incomplete VKH disease 27 (19.28\%) with neurological findings and 14 (10\%) with dermatological findings (Figure 3).

Of the 40 patients considered not having $\mathrm{VKH}$, the disease was unilateral in 4 patients $(2.85 \%)$, previous ocular trauma (surgical or not) was present in 3 patients $(2.14 \%)$, presence of another ocular or systemic disease in 5 patients $(3.58 \%)$, fundoscopy examination was impossible, due to posterior synechiae in 22 patients $(15.71 \%)$ and 7 patients $(5 \%)$ simply did not fulfill the criteria for the diagnosis of VKH disease (Figure 4).

Complete Vogt-Koyanagi-Harada disease (criteria 1 to 5 must be present)

1. No history of penetrating ocular trauma or surgery preceding the initial onset of uveitis.

2. No clinical or laboratory evidence suggestive of other ocular disease entities.

3. Bilateral ocular involvement ( $a$ or $b$ must be met, depending on the stage of disease when the patient is examined).

a. Early manifestations of disease. (1) There must be evidence of a diffuse choroiditis (with or without anterior uveitis, vitreous inflammatory reaction, or optic disk hyperemia), which may manifest as one of the following:

(a) Focal areas of subretinal fluid, or

(b) Bullous serous retinal detachments.

(2) With equivocal fundus findings; both of the following must be present as well: (a) Focal areas of delay in choroidal perfusion, multifocal areas of pinpoint leakage, large placoid areas of hyperfluorescence, pooling within subretinal fluid, and optic nerve staining (listed in order of sequential appearance) by fluorescein angiography, and (b). Diffuse choroidal thickening, without evidence of posterior scleritis by ultrasonography.

b. Late manifestations of disease. (1) History suggestive of prior presence of findings from $3 a$, and either both (2) and (3) below, or multiple signs from

(3): (2) Ocular depigmentation (either of the following manifestations is sufficient): (a) Sunset glow fundus, or (b) Sugiura sign.

(3) Other ocular signs: (a) Nummular chorioretinal depigmented scars, or (b) Retinal pigment epithelium clumping and/or migration, or (c) Recurrent or chronic anterior uveitis.

4. Neurological/auditory findings (may have resolved by time of examination).

a. Meningismus (malaise, fever, headache, nausea, abdominal pain, stiffness of the neck and back, or a combination of these factors; headache alone

is not sufficient to meet definition of meningismus, however), or

b. Tinnitus, or

c. Cerebrospinal fluid pleocytosis.

5. Integumentary finding (not proceding onset of central nervous system or ocular disease).

a. Alopecia, or

b. Poliosis, or

c. Vitiligo,

Incomplete Vogt-Koyanagi-Harada disease (criteria 1 to 3 and either 4 or 5 must be present)

1. No history of penetrating ocular trauma or surgery preceding the initial onset of uveitis, and

2. No clinical or laboratory evidence suggestive of other ocular disease entities, and

3. Bilateral ocular involvement.

4. Neurologic/auditory findings; as defined for complete Vogt-Koyanagi-Harada disease above, or

5. Integumentary findings; as defined for complete Vogt-Koyanagi-Harada disease above.

Probable Vogt-Koyanagi-Harada disease (isolated ocular disease; criteria 1 to 3 must be present)

1. No history of penetrating ocular trauma or surgery preceding the initial onset of uveitis.

2. No clinical or laboratory evidence suggestive of other ocular disease entities.

3. Bilateral ocular involvement as defined for complete Vogt-Koyanagi-Harada disease above.

Figure 1 - 2001 Revised diagnostic criteria for Vogt-Koyanagi-Harada disease ${ }^{(9)}$ 


\section{DISCUSSION}

Patients who have been previously diagnosed with VKH disease were reclassified according to the revised diagnostic criteria proposed by the VKH disease committee in 2001. Of the 140 patients, VKH disease diagnosis was confirmed in 99 of them (70.71\%). Among the patients in which VKH was excluded, $22(53.65 \%)$ had no fundoscopy records in their medical chart. Probably those patients had a late diagnosis, during the convalescent phase of the disease.

Some authors studied the application of the revised diagnostic criteria in Japanese patients. Forty-nine patients were retrospectively studied considering early and late phases of the disease and a difference between the 2 phases was found. In this study $6 \%$ of the patients were considered not to have VKH disease according to the revised criteria.

In another Japanese study, Kitamura et al. compared two different criteria for VKH disease, the revised diagnostic criteria and Sugiura's diagnosis system. The authors studied 169 patients, all of them Japanese and 56\% female. The group of patients was very homogeneous ${ }^{(11)}$.

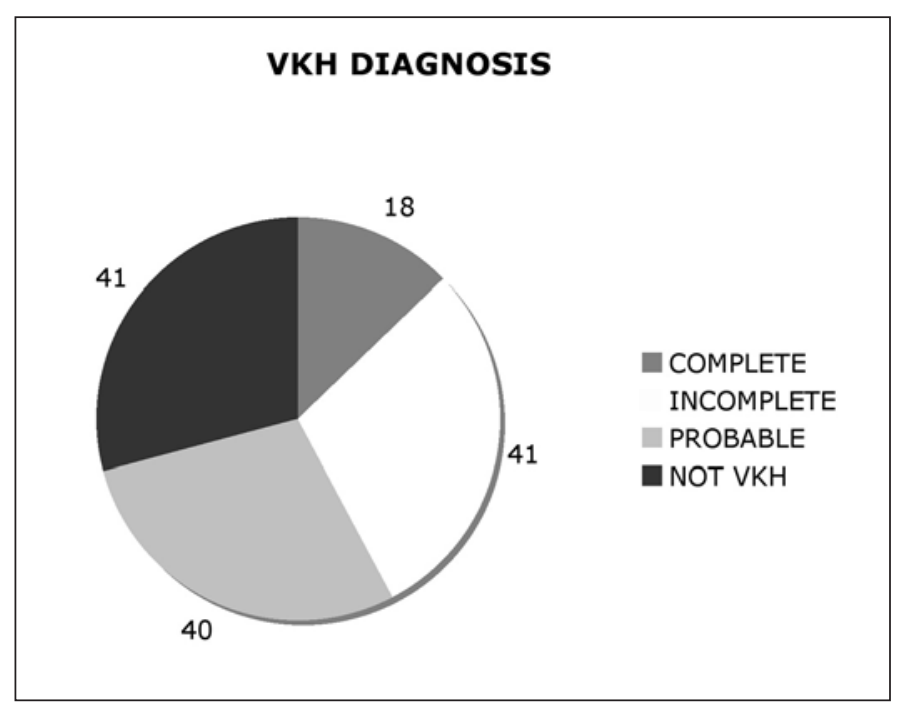

Figure 2 - Patient distribution by revised diagnostic criteria

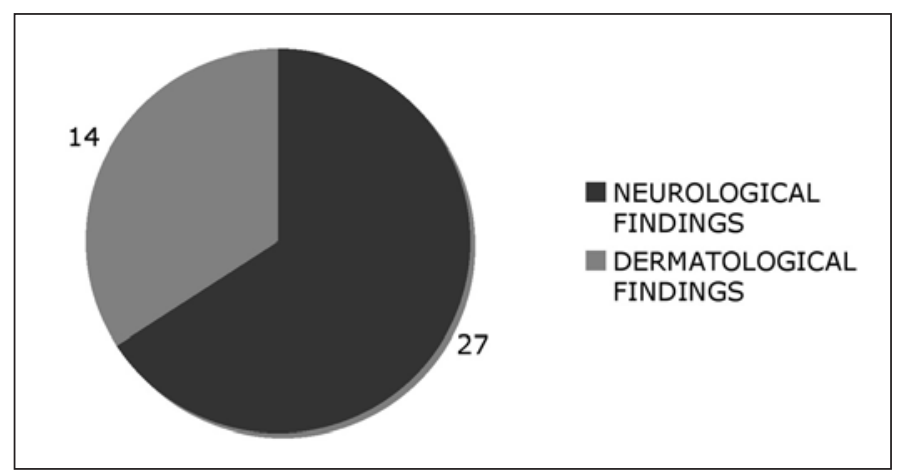

Figure 3 - Incomplete disease

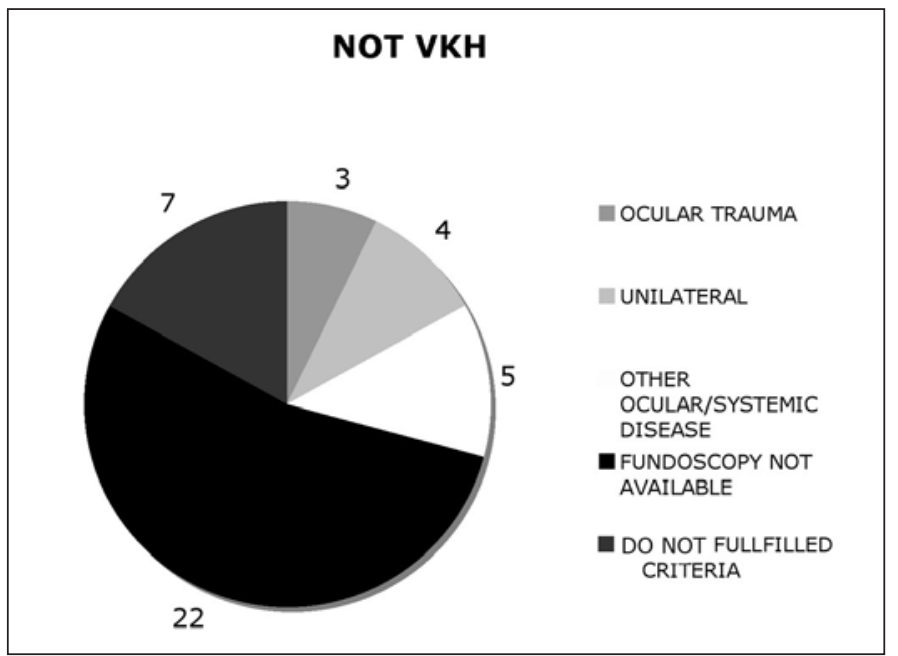

Figure 4 - Reasons for not considering VKH disease

In Brazil, due to miscegenation it is very difficult to have a group of patients with homogeneous racial characteristics. Out of the 169 patients, $91.7 \%$ were considered as having VKH disease according to the 2 criteria, $8.3 \%$ were excluded on the basis of the revised diagnostic criteria. In conclusion, the study document shows that the revised criteria are capable to make a highly sensitive and specific VKH disease diagnosis.

In the present study, 29.9\% of the patients did not fulfill the diagnosis of VKH disease due to the revised criteria. If we exclude the patients whose medical charts were incomplete, those that do not have the fundoscopy data, $16.1 \%$ of the patients did not fulfill the diagnosis of VKH disease according to the revised criteria.

In this study the accordance of the former and the new diagnosis criteria is different from that which was found in the 2 Japanese studies ${ }^{(10-11)}$. A probable explanation for this fact could be related to the homogeneity of the groups and maybe because the Japanese studies considered different stages of the disease.

\section{CONCLUSION}

However, the authors consider that the revised diagnostic criteria have good sensitivity and specificity and are very useful and applicable to help in the diagnosis of VKH disease.

\section{RESUMO}

Objetivo: Determinar a aplicabilidade do "Internacional revised diagnostic criteria for Vogt-Koyanagi-Harada disease". Métodos: Estudo retrospectivo. Prontuários de 140 pacientes com diagnóstico de "Vogt-Koyanagi-Harada disease", do setor de uveítes da Universidade Federal de São Paulo (UNIFESP), foram revisados e classificados segundo o "Internacional revised diagnostic criteria". Resultados: Dos 140 pacientes, 
$12,85 \%$ preencheram os critérios para doença completa, $29,28 \%$ doença incompleta, $28,57 \%$ "provável" doença de Vogt-Koyanagi-Harada e 28,27\% foram consideradas como não sendo doença de Vogt-Koyanagi-Harada. Conclusão: Os autores consideraram que o "International revised diagnostic criteria" tem boa aplicabilidade e é muito útil no diagnóstico da doença de Vogt-Koyanagi-Harada.

Descritores: Síndrome uveomeningoencefálica/diagnóstico; Técnicas de diagnóstico oftalmológico; Uveíte; Granuloma

\section{REFERENCES}

1. Read RW, Rao NA, Cunningham ET. Vogt-Koyanagi-Harada disease. Curr Opin Ophthalmol. 2000;11(6):437-42. Review.

2. Rao NA. Mechanisms of inflammatory response in sympathetic ophthalmia and VKH syndrome. Eye. 1997;11(Pt 2):213-6.
3. Sheu SJ. Update on uveomeningoencephalitides. Curr Opin Neurol. 2005; 18(3):323-9.

4. Gocho K, Kondo I, Yamaki K. Identification of autoreactive T cells in VogtKoyanagi-Harada disease. Invest Ophthalmol Vis Sci. 2001;42(9):2004-9.

5. Moorthy RS, Inomata H, Rao NA. Vogt-Koyanagi-Harada syndrome. Surv Ophthalmol. 1995;39(4):265-92. Review.

6. Bykhovskaya I, Thorne JE, Kempen JH, Dunn JP, Jabs DA. Vogt-KoyanagiHarada disease: clinical outcomes. Am J Ophthalmol. 2005;140(4):674-8.

7. Andrade RE, Muccioli C, Farah ME, Nussenblatt RB, Belfort R Jr. Intravitreal triamcinolone in the treatment of serous retinal detachment in Vogt-KoyanagiHarada syndrome. Am J Ophthalmol. 2004;137(3):572-4.

8. Taylor S, Lightman S. Recurrent anterior uveitis in patients with VogtKoyanagi-Harada syndrome. Arch Ophthalmol. 2004;122(6):922-3.

9. Read RW, Holland GN, Rao NA, Tabbara KF, Ohno S, Arellanes-Garcia L, et al. Revised diagnostic criteria for Vogt-Koyanagi-Harada disease: report of an international committee on nomenclature. Am J Ophthalmol. 2001;131(5): 647-52.

10. Yamaki K, Hara K, Sakuragi S. Application of revised diagnostic criteria for Vogt-Koyanagi-Harada disease in Japanese patients. Jpn J Ophthalmol. 2005; 49(2):143-8.

11. Kitamura M, Takami K, Kitachi N, Namba K, Kitamei H, Kotake S, et al. Comparative study of two sets of criteria for the diagnosis of Vogt-KoyanagiHarada's disease. Am J Ophthalmol. 2005;139(6):1080-5.

\title{
X Congresso Internacional de Catarata e Cirurgia Refrativa
}

\section{Congresso Internacional de Administração em Oftalmologia}

\author{
14 a 17 de maio de 2008 \\ Centro de Convenções de Goiânia \\ Goiânia - GO
}

\author{
INFORMAÇÕES \\ JDE Comunicação e Eventos \\ Tels.: (11) 5084-9174/5082-3030 • Fax: (11) 5574-8261 \\ E-mail: jdecomev@uol.com.br \\ home page: www.catarata-refrativa.com.br
}

